\title{
Monitoreo Terapéutico de Fármacos y Farmacocinética
}

\author{
Therapeutic Monitoring of Drugs and Pharmacokinetics
}

Ana Luisa Robles Piedras ${ }^{\text {. }}$

\begin{abstract}
:
Therapeutic drug monitoring (TDM) is a tool that can guide the clinician to provide an effective and safe drug therapy in the individual patient. It is a team work tool. The clinical pharmacist plays an important role to guide the team conformed by doctors, nurses and laboratory professionals. TDM involves not only measuring drug concentrations, but also the clinical interpretation of the result. This requires knowledge of the pharmacokinetics, sampling time, drug history, the patient's clinical condition and specific laboratory results.
\end{abstract}

\section{Keywords:}

Therapeutic, drug, monitoring, pharmacokinetics

\section{Resumen:}

El Monitoreo Terapéutico de Fármacos (TDM) es una herramienta que puede guiar al médico para proporcionar una terapia farmacológica efectiva y segura a un paciente de manera individualizada. Es una herramienta de trabajo en equipo. El farmacéutico clínico desempeña un papel importante para guiar al equipo conformado por médicos, enfermeras y profesionales de laboratorio. El TDM implica no solo medir las concentraciones del fármaco, sino también la interpretación clínica del resultado. Esto requiere conocimiento de la farmacocinética, tiempo de muestreo, historial de medicación, el estado clínico del paciente y los resultados de laboratorio específicos.

\section{Palabras Clave:}

Monitoreo, terapéutico, fármacos, farmacocinética

\section{Introducción}

La gran mayoría de fármacos son manejados de una manera muy estándar. Los fármacos son dosificados por unidad de peso, por ejemplo, $\mathrm{mg} / \mathrm{Kg}$ de peso corporal, y después la dosis es ajustada basándose en la respuesta clínica, según la evaluación empírica del médico. Esto a menudo es descrito como "valoración al efecto clínico". Para un pequeño subconjunto de fármacos, los efectos clínicos (farmacodinamia) pueden ser evaluados de manera objetiva, a través de mediciones de laboratorio, como, por ejemplo, las estatinas y los niveles de lípidos en sangre. Pero para un subgrupo de fármacos aún más pequeño, la relación farmacocinética/farmacodinamia no es predecible a partir de la dosis administrada, y la farmacocinética es altamente variable entre los individuos, por lo que, para estos fármacos, su manejo clínico puede ser particularmente desafiante. Es para estos fármacos en los que la aplicación del monitoreo terapéutico es más efectiva.

\section{Monitoreo Terapéutico de Fármacos}

El Monitoreo Terapéutico de Fármacos (TDM, por sus siglas en inglés) es una rama de la farmacología clínica y la farmacia clínica, que se especializa en la medición de las concentraciones de fármacos en fluidos biológicos, principalmente suero, plasma o sangre.

La Asociación Internacional para el Monitoreo Terapéutico de Fármacos y Toxicología Clínica adoptó la siguiente definición para el monitoreo de fármacos: 1

"La monitorización terapéutica de fármacos se define como la medición realizada en el laboratorio de un parámetro que, con la interpretación adecuada, influirá directamente en los procedimientos de prescripción. Comúnmente, la medición está en una matriz biológica de un xenobiótico prescrito, pero también puede ser de un

Autor de Correspondencia, Universidad Autónoma del Estado de Hidalgo, https://orcid.org/0000-0002-8697-605X, Email: roblesa@uaeh.edu.mx 
compuesto endógeno prescrito como terapia de reemplazo en un individuo que tiene deficiencia fisiológica o patológica en ese compuesto".

Aunque el TDM puede servir como una guía práctica para aclarar varias condiciones a una cierta respuesta clínica inesperada en tratamientos crónicos con medicamentos, su objetivo principal es la optimización y/o individualización de los regímenes de dosificación y el lograr una terapia farmacológica eficaz y segura al mantener las concentraciones de fármaco, dentro de un rango objetivo (rango terapéutico, ventana terapéutica, intervalo terapéutico). La ventana terapéutica se define generalmente como el rango (es decir, valores de referencia superiores e inferiores) de las concentraciones plasmáticas de fármacos que producen efectos terapéuticos con un riesgo mínimo de reacciones adversas (también denominados efectos secundarios o tóxicos) en la mayoría de los pacientes.

El objetivo del TDM es mantener las concentraciones plasmáticas de los fármacos dentro de un intervalo predeterminado para optimizar los resultados del tratamiento, ya que las concentraciones por encima de los valores de referencia, generalmente están vinculadas a una mayor probabilidad de que se presenten en el paciente mayores efectos secundarios, mientras que las que están por debajo de los valores de referencia puedan dar como consecuencia, la ineficacia o una respuesta insatisfactoria del tratamiento farmacológico.

La concentración de un fármaco en el sitio de acción no puede medirse de manera rutinaria, pero se ha demostrado que los efectos adversos o deseados pueden correlacionarse mejor con las concentraciones plasmáticas o sanguíneas, que con la dosis. Para algunos fármacos, las mediciones de concentración son un sustituto valioso de la exposición a éste, particularmente si no hay manera sensible de medir el efecto.

Cuando hay una gran variabilidad interindividual entre la dosis administrada y el efecto, por ejemplo, cuando hay una gran variación farmacocinética, es difícil individualizar la dosis del fármaco; esto es particularmente relevante para aquellos fármacos que presentan un margen terapéutico estrecho o una farmacocinética dependiente de la concentración. 2

De igual manera, en terapias de tipo crónico, por una variedad de razones, con el paso del tiempo en algunos fármacos pueden presentarse variaciones dentro del mismo individuo, y en estos casos, el TDM puede resultar sumamente útil.

Las situaciones en las que el monitoreo farmacocinético puede ser útil e indicado son las siguientes: 3
- El fármaco exhibe una ventana terapéutica estrecha, en la que la dosis que produce un efecto clínico beneficioso es cercana a la dosis que probablemente produzca un efecto adverso.

- No existe una relación de respuesta a la dosis que sea predecible, de tal manera que una dosis dada que produce un efecto beneficioso en un individuo, puede producir un efecto adverso en otro.

- La concentración del fármaco en sangre, no se puede predecir a partir de la dosis, debido a la variabilidad inter e intraindividual de los niveles plasmáticos.

- La eficacia y la toxicidad de un fármaco se correlacionan con la concentración sérica y existe una mejor correlación entre la concentración de fármaco libre que con que la concentración total del fármaco.

- Existen indicaciones clínicas para la monitorización terapéutica del fármaco, por ejemplo, mala respuesta al fármaco, sospecha de fracaso del tratamiento debido al incumplimiento o signos de toxicidad a pesar de no haber ajuste de dosis.

- Cuando existe dificultad para interpretar los niveles terapéuticos o de baja toxicidad de un fármaco basándose solo en la evidencia clínica y no existe un parámetro clínico claramente definido para el ajuste de la dosis.

Por lo general, los fármacos anticonvulsivos, los fármacos cardioactivos, los inmunosupresores, los antiasmáticos, los antidepresivos, los antirretrovirales, los antineoplásicos y los antibióticos con ventanas terapéuticas estrechas, como la vancomicina y los aminoglucósidos, requieren una monitorización terapéutica de rutina. En la mayoría de los casos, la concentración mínima (15 a 30 minutos antes de la siguiente dosis), ya sea en sangre total, plasma o suero, es el espécimen preferido para la monitorización farmacocinética (TDM), excepto para ciertos antibióticos (vancomicina y aminoglucósidos), donde se controlan los niveles máximos y mínimos (predosis y posdosis); la vancomicina y los aminoglucósidos pueden producir nefrotoxicidad y ototoxicidad graves. 4

\section{Beneficios del TDM}

Los beneficios que se obtienen al realizar la monitorización farmacocinética, son varios, y desde hace varias décadas, han sido demostrados en varios países. Entre ellos se encuentran: 5 
- Identificación de falta de cumplimiento por parte del paciente. Si tras la medición por parte del laboratorio se reporta que el nivel de fármaco es cero o subterapéutico, o que el nivel del fármaco es altamente variable, son indicaciones de incumplimiento total o parcial. Cabría analizar también, si acaso el nivel subterapéutico se deba a que el paciente sea un metabolizador ultrarrápido.

- Individualización de dosis. El metabolismo y la eliminación de ciertos fármacos dependen de varias condiciones fisiopatológicas, tales como la uremia y la enfermedad hepática. En los pacientes de edad avanzada, los niños y en las mujeres embarazadas, es necesaria la participación de los farmacéuticos para que se realice el ajuste de dosis de ciertos fármacos basándose en el TDM.

- Mejora en la seguridad del paciente y ayuda a la disminución de la estancia hospitalaria. El TDM puede mejorar en gran medida la seguridad del paciente a través de la individualización la dosis que propicie a una mejoría más pronta y así, disminuir la estancia hospitalaria, lo que ahorra costos de atención médica.

- Investigación de no respuesta en el paciente. Si un paciente no responde a un medicamento, también puede deberse a una absorción deficiente o una variación genética en el metabolismo del fármaco. En estas circunstancias, el TDM es útil para identificar a estos pacientes. Si la concentración del fármaco está dentro del rango terapéutico, entonces un paciente puede no responder a ese fármaco.

\section{Conclusión}

Para lograr el éxito de una monitorización terapéutica de fármacos, es esencial una buena comunicación entre médicos, enfermeras, profesionales de laboratorio y farmacéuticos, pues el TDM no sólo implica medir las concentraciones, sino también realizar una adecuada interpretación clínica del resultado. Esto requiere conocer la farmacocinética, el tiempo correcto al cual debe tomarse la muestra, el historial farmacológico y la condición clínica del paciente.

\section{Referencias}

[1] Touw DJ, Neef C, Thomson AH, Vinks AA, Cost-Effectiveness of Therapeutic Drug Monitoring Committee of the International Association for Therapeutic Drug Monitoring and Clinical Toxicology. Cost-effectiveness of therapeutic drug monitoring: a systematic review. Ther. Drug Monit. 2005; 27(1): 10-17.

[2] Gross AS. Best practice in therapeutic drug monitoring. Br. J. Clin. Pharmacol. 2001; 52 Suppl 1: 5S-10S.
[3] Kang JS, Lee MH. Overview of therapeutic drug monitoring. Korean J. Intern. Med. 2009; 24(1): 1-10.

[4] Roberts JA, Norris R, Paterson DL, Martin JH. Therapeutic drug monitoring of antimicrobials. Br. J. Clin. Pharmacol. 2012; 73(1): 2736.

[5] Gogtay NJ, Kshirsagar NA, Dalvi SS. Therapeutic drug monitoring in a developing country: an overview. Br. J. Clin. Pharmacol. 2001; 52 Suppl $1: 103 S-108$ S. 